\title{
Acoustic signals of rotating drums generated based on DEM simulations
}

\author{
Yaoyu $\mathrm{Li}^{1}$, Jie $\mathrm{Bao}^{2}$, Runyu Yang ${ }^{1 *}$ \\ ${ }^{1}$ School of Materials Science \& Engineering, University of New South Wales, Sydney, 2052, Australia \\ ${ }^{2}$ School of Chemical Engineering, University of New South Wales, Sydney, 2052, Australia
}

\begin{abstract}
Acoustic emission (AE) or vibration signal has been applied in detecting operations of grinding mills in many industries. This paper proposes an approach to generate AE signals based on the particle-wall impacts. Through a combination of multi-mode vibrations and the calibration of the key parameters, the model was able to reproduce experimental data. The AE model was then implemented into a discrete element method (DEM) modelling of particle flow in a rotating mill. The AE signals of the mill under different filling levels and rotation speeds were generated and analysed, mainly focusing on the frequency and magnitude of each vibration mode. The link between the AE signals and the particle-wall impact energy was explored.
\end{abstract}

\section{Introduction}

Milling is an important size reduction process in many industries, such as mineral and coal industries $[1$, 2]. It is an energy-intensive process in which only $4 \sim 8 \%$ of the total input energy is utilised for particle breakage. Thus it is essential that grinding circuits are properly designed and operated as efficiently as possible.

Direct access of internal information of industrial mills is challenging because of the harsh internal environment which makes internal installation of any online measuring devices impractical [3]. Monitoring mill operation in industries is often achieved by attaching vibration sensors (e.g., wireless accelerometer sensor) on the mill surface to obtain mechanical/acoustic vibration signal $[4,5]$.

Research has been conducted to establish the link between the $\mathrm{AE}$ signal and internal particle flow dynamics. Zeng and Forssberg [6] used AE signal to predict the power draw, feed rate, particle size distributions and pulp densities of wet mills . Tang et al. $[2,7,8]$ analysed vibration signals in a wet ball mill and divided the signals into three parts, corresponding to (from low to high) natural frequency, main impact frequency and secondary impact frequency bands. More recently, they developed a modelling strategy based on both vibration and acoustic signals [9]. However, such link, which is based on the black-box observation, is implicit as the internal flow is still unknown to experiments. In addition, AE signals have only been used to link to macroscopic properties of flow such as power draw and filling level. More effort is required to link detailed dynamics such as energy distribution inside mills and particle size distribution with AE signals.

Numerical modelling based on the discrete element method (DEM) can establish the link between the information collected on mill walls with internal particle flows. Through DEM simulations, McElory et al. [10] used the particle-wall collision energy data to predict particle flow regimes in a rotating drum. They also established a link between particle-wall collision energy with particle-particle collision energy [11]. To generate information more readily measurable in experiments, Hosseini et al. [4] simulated the AE signals in a tumbling mill based on the impact force and system response. They established a system response function to an impact which was then implemented into DEM simulations. The generated $\mathrm{AE}$ signals were compared with experimental measurement. However, they did not conduct more detailed analysis on the frequencies of the signals and link the signals to the particle flow in the mill.

In this work, we developed an AE signal model based on particle-wall collisions. The model was calibrated with the literature data obtained from the experiments of single particle impact test. The AE model was then implemented into the DEM simulation to generate AE signals of a rotating drum with different filling levels and rotation speeds. Finally, the characteristics of the signals were analysed and linked to particle-wall impact energy.

\section{AE model and model calibration}

\subsection{Acoustic emission model}

In the proposed $\mathrm{AE}$ model, the vibrational displacement of a plate induced by an impact from a particle is approximated as [12]:

$$
U(x, t)=\delta(x) \sum_{i=1}^{m} u_{i}(t)
$$

\footnotetext{
* Corresponding author: r.yang@unsw.edu.au

A video is available at https://doi.org/10.48448/1k3w-y947
} 
where $m$ is the total number of vibration modes, $u_{i}(t)$ is the vibration displacement of the vibration mode $i$ of the impact point at time $\mathrm{t}$, and $\delta(x)=\exp (-\alpha x)$ is a function describing an exponential decay of the vibration from the impact point to a location with distance $x$ where the signal is collected [13]. $\alpha$ is the spatial attenuation coefficient.

The vibration displacement of vibration mode $i$ is approximated as a damped harmonic motion given by:

$$
u_{i}(t)=u_{i, \max } e^{-\beta t} \sin \left(2 \pi f_{i} t+\varphi_{i}\right)
$$

where $\beta$ is the attenuation coefficient, $f_{i}$ is the frequency (Hz) of vibration mode $i$ and $\varphi_{i}$ is the phase. $u_{i, \max }$ is the maximum amplitude. It is linked to impact force or impact kinetic energy $[14,15]$ which can be obtained from Hertzian contact model.

As acoustic pressure is often measured in experiments [16], a parameter $\varepsilon$ is adopted to link displacement in Eq. (1) with pressure given by

$$
P(x, t)=\varepsilon U(x, t)=\varepsilon \delta(x) \sum_{i=1}^{m} u_{i}(t)
$$

The model involves several assumptions: an impact is regarded as an instant event; the impact energy only affects vibration amplitude but not frequency and phase $[14,15]$; small impact angles has little effects on $\mathrm{AE}$ signals [15]; and friction has no effect on AE signals.

\subsection{Parameter calibration}

The parameters in Eq. (3), including pressure term $\varepsilon u_{\text {max }}$, frequency $f$ and $\varphi$, were calibrated based on a pendulum impact experiment conducted by Takahagi et al. [16]. In the experiment, a steel ball with diameter 12.7 $\mathrm{mm}$ collided with a steel plate at the velocity of $1.2 \mathrm{~m} / \mathrm{s}$, and the sound signals were collected by a microphone near the surface of the plate.

Fig. 1a shows the measured sound signal. To fit the experimental results, six modes of vibration were adopted to reduce computing cost while still retain the key features of the signals. As shown in Fig. 1a, the fitting result is able to capture the signal patterns of the experiment. Table 1 shows the parameters calibrated through the fitting. It should be noted that as only six vibration modes were considered in the fitting, not all features of the experimental signals were reproduced in the generated AE signals. A higher-fidelity reproduction of the signals would require more vibration modes.

Table 1. AE model parameters in the simulations

\begin{tabular}{ccll}
\hline Mode No. & $\varepsilon u_{\max }(\mathrm{Pa})$ & $f(\mathrm{kHz})$ & $\varphi$ \\
\hline 1 & 4.05 & 5.81 & 2.24 \\
2 & 3.38 & 7.18 & 1.79 \\
3 & 7.17 & 11.6 & 3.13 \\
4 & 5.68 & 13.5 & -2.48 \\
5 & 2.63 & 15.9 & 1.71 \\
6 & 5.61 & 21.3 & -1.62 \\
\hline
\end{tabular}

To capture the temporal decay of the AE signals, experimental measurement of the signals over a long period was shown in Fig. 1b. The signal shows an exponential decay and almost disappear over $50 \mathrm{~ms}$. By setting temporal attenuation coefficient $\beta=57$, the simulate result was comparable with experimental data. The spatial attenuation coefficient $\alpha$ was selected such that the signal decays by half every $50 \mathrm{~mm}$.
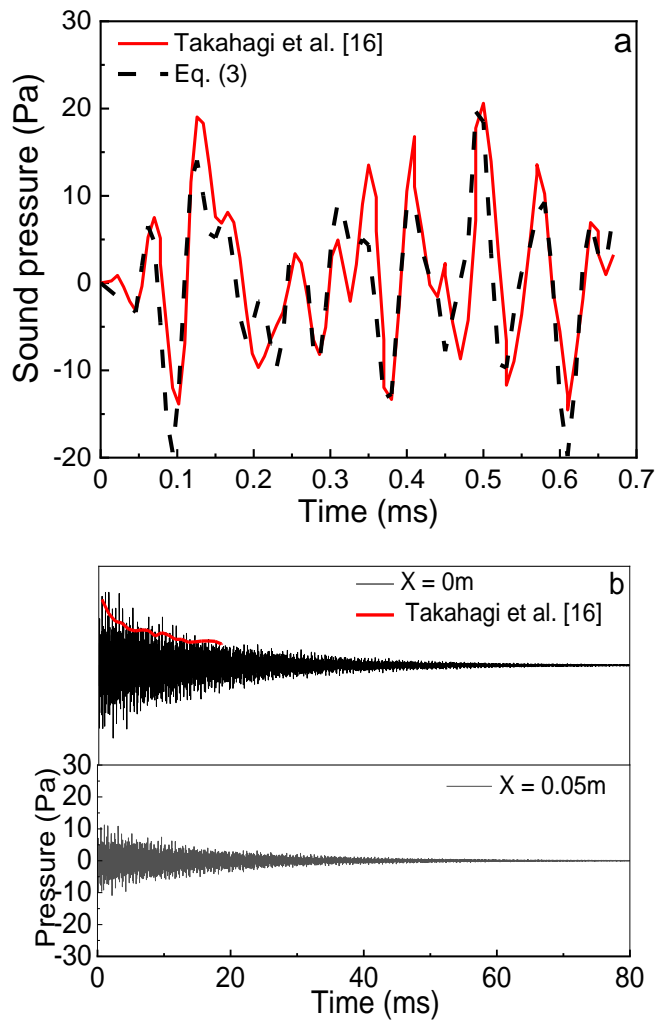

Fig. 1 Acoustic emission signals of steel particle impact with steel plate experiment [16] ( - ) and acoustic model (--): (a) short time scale; (b) long time scale of different sensor locations.

\section{DEM modelling}

\subsection{DEM model and parameters}

The governing equations of DEM model based on Newton second law for the translational and rotational motion of particle $i$ with mass $m_{\mathrm{i}}$ and moment of inertia $I_{\mathrm{i}}$ can be written as:

$$
\begin{aligned}
& m_{i} \frac{d \mathbf{v}_{i}}{d t}=m_{i} \mathbf{g}+\sum_{j}\left(\mathbf{F}_{i j}^{n}+\mathbf{F}_{i j}^{s}\right) \\
& I_{i} \frac{d \boldsymbol{\omega}_{i}}{d t}=\sum_{j}\left(\mathbf{R}_{i} \times \mathbf{F}_{i j}^{S}-\mu_{r} R_{i}\left|\boldsymbol{F}_{i j}^{n}\right| \boldsymbol{\omega}_{i}\right)
\end{aligned}
$$

where $\mathbf{v}_{i}, \omega_{i}$ and $I_{i}$ are, respectively, the translational velocity, angular velocity and moment of inertia of the particle, $\mu_{r}$ the rolling friction coefficient. $\boldsymbol{R}_{i}$ is a vector running from the centre of the particle to the contact point with its magnitude equal to particle radius $R_{i} . \mathbf{F}_{i j}^{n}$ and $\mathbf{F}_{i j}^{S}$ represent, respectively, the normal contact force and the tangential contact force imposed on particle $i$ by particle $j$ using Hertz-Mindlin contact model [17].

In this work, a simulation started with a horizontal drum partially filled by mono-sized spherical particles. Periodic boundary condition was applied along the axial direction to avoid wall effect. After a stable packed bed was formed, the drum rotated at a prescribed speed and the flow properties were anlaysed when the flow reached 
a steady state (by monitoring particle velocity and power draw).

Table 2 shows the operation parameters in the simulations. In the current study, rotation speed and filling level were varied to generate different particle flow patterns.

Table 2. Operation parameters in the simulation

\begin{tabular}{cc}
\hline Parameter & Value \\
\hline Particle size, $d(\mathrm{~mm})$ & 12.7 \\
Filling level, $F L$ & $0.35,0.5$ \\
Particle density, $\rho\left(\mathrm{kg} / \mathrm{m}^{3}\right)$ & $7.8 \times 10^{3}$ \\
Drum diameter, $\mathrm{D}(\mathrm{mm})$ & 635 \\
Young's modulus, $E\left(\mathrm{~N} / \mathrm{m}^{2}\right)$ & $1.5 \times 10^{10}$ \\
Sliding friction coefficient, $\mu$ & 0.3 \\
Rolling friction coefficient, $\mu_{r}$ & $1.0 \times 10^{-6}$ \\
Poisson ratio, $\gamma$ & 0.27 \\
Normal damping coefficient & $1.0 \times 10^{-6}$ \\
Rotation speed, $\mathrm{N}(\mathrm{rpm})$ & $30-75$ \\
\hline
\end{tabular}

Fig. 2 shows particle flow pattern at filling level 0.50 and rotating speed $45 \mathrm{rpm}$. The colour represents particle velocity. The flow pattern is a typical cascading regime as a clear ' $S$ ' shape flow [18]. The outermost layer of the particle flow has larger particle velocity than the inner part.

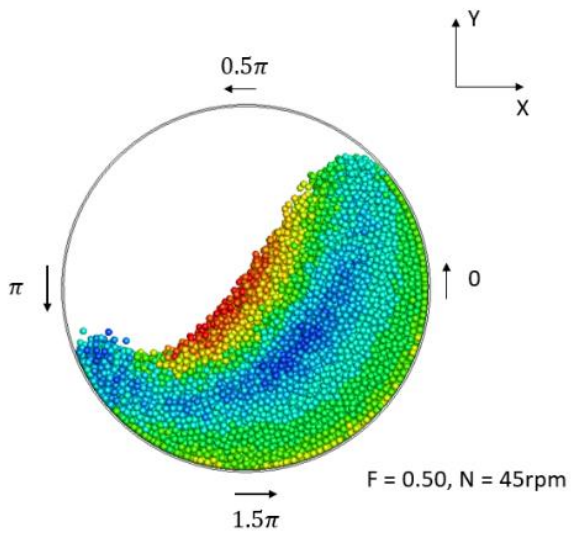

Fig. 2 Particle flow in the rotating drum (particle color represents the magnitude of velocity).

After the flow has reached a steady state at $4 \mathrm{~s}$, then every particle-wall collision over a period of $0.2 \mathrm{~s}$ were recorded and analysed. The collision energy is defined as $E=\frac{1}{2} m v_{n, i j}^{2}$ where $m$ is particle mass and $v_{n, i j}$ is the normal component of the relative velocity between the particle and the wall at the time of the collision.

Fig. 3a shows the accumulative distributions of the particle-wall impact energy at different rotation speeds. As the rotation speed increases, the distributions line shifts to the right. As the mill is dominated by impacts with large impact energy and to quantify the collision energy with a single index, $\mathrm{E}_{80}$ (energy larger than $80 \%$ of collision energy) was adopted. Fig. $3 \mathrm{~b}$ shows $\mathrm{E}_{80}$ increases almost linearly with rotation speed when FL is 0.35 . More specifically, $\mathrm{E}_{80}$ when FL is 0.35 increases more quickly than when FL is 0.50 . After $60 \mathrm{rpm}, \mathrm{E}_{80}$ with $\mathrm{FL}=0.50$ increases little because the flow regime is transmitted to the cataracting regime.

\section{AE signals of the drum}

The AE signal was calculated based on the impact information including impact energy, impact time, and impact positions from DEM simulations in section 3. The signal collected by the sensor at a given time is the linear combination of all the impacts before the given time. In addition, the original signal caused by impacts decays with a distance as defined by Eq. (3), so the impacts too far away from the sensor or too long $(50 \mathrm{~ms})$ before the collection time were not included in the calculation.
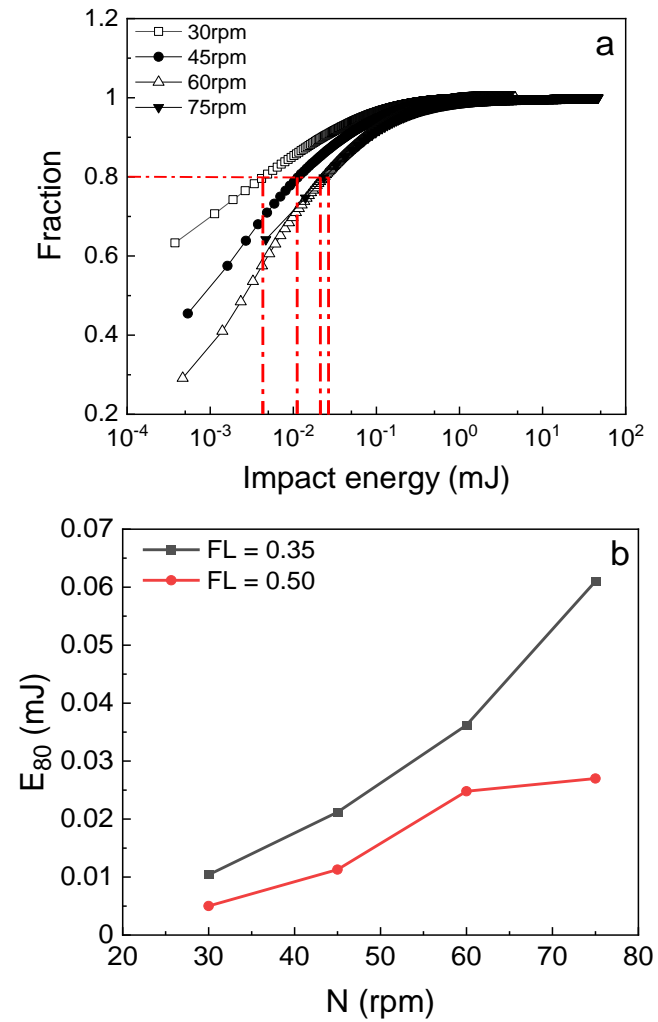

Fig. 3 (a) Accumulated distributions of particle-wall impact energy; and (b) variations of $\mathrm{E}_{80}$ with rotation speeds at different filling levels.

Fig. 4a shows the AE signal obtained from a sensor fixed at the bottom of the drum. The signals show a typical sound pattern. But as the flow is at a steady state, the signal pattern is relative stable without sharp fluctuations.

Fig. $4 \mathrm{~b}$ is the AE signal in frequency domain through Fast Fourier Transform (FFT) analysis. The sampling frequency is $10^{5} \mathrm{~Hz}$ to follow the Nyquist-Shannon sampling theorem which requires the sampling frequency of a signal is at least double the frequency of its highest frequency component. It can be found that there are six main signal peaks $\left(\mathrm{P}_{1}-\mathrm{P}_{6}\right)$ in frequency spectrum. The amplitude of the main peaks depends on the flow behaviours which is different at different operational conditions.

As both particle-wall impact energy and AE signal can be used to characterise particle flow, there may be an underlying statistical relation between the peaks of the $\mathrm{AE}$ signal and impact energy $\mathrm{E}_{80}$, which can be given by:

$$
E_{80}=\sum_{i} w_{i} P_{i}
$$

where $P_{i}$ is the peak in Fig. $4 \mathrm{~b}$ and $w_{i}$ is the coefficient with unit $\mathrm{J} / \mathrm{Pa}$.

Fig. 5 shows the predicted $\mathrm{E}_{80}$ from Eq. (6) are quite comparable with $\mathrm{E}_{80}$ from the DEM simulations, 
indicating that there exists a linear relation between $\mathrm{AE}$ signal and the impact energy in the current system. The finding suggests that the signals obtained from the drum surface can be linked to the energy distribution of particle flow. However, the relation in Eq. (6) is only applicable to the mono-sized particles. More research is required to establish the relation for systems with multi-sized particles.
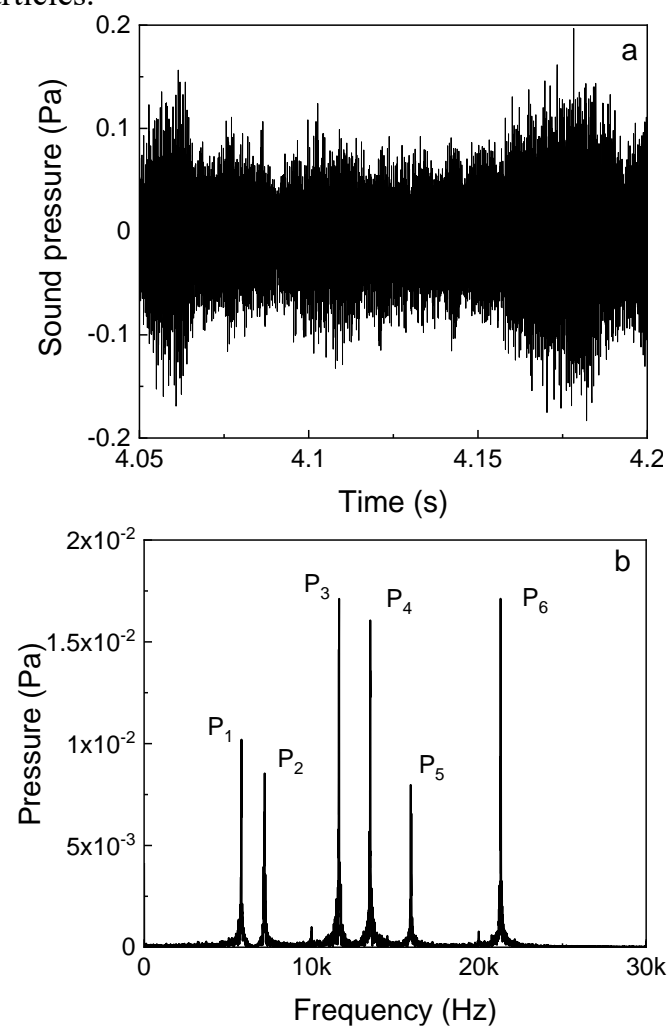

Fig. 4 Simulated AE signals of the drum $(F L=0.50, N=$ $45 \mathrm{rpm}$ ): (a) time domain; and (b) frequency spectrum.

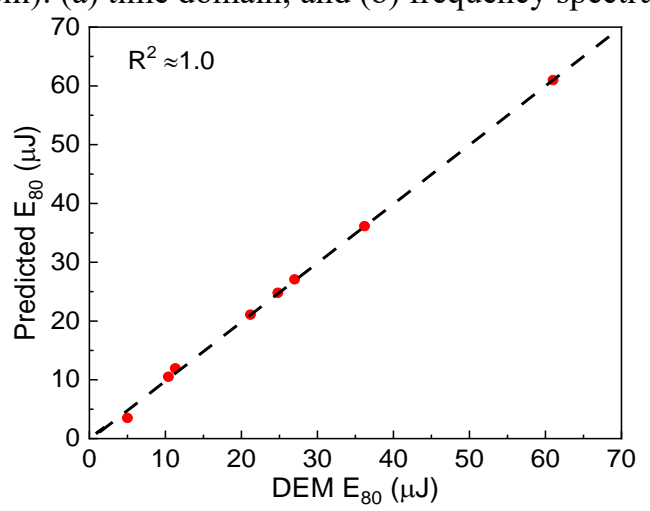

Fig. 5 Comparisons of predicted $\mathrm{E}_{80}$ from Eq. 6 and the simulated $\mathrm{E}_{80}$

\section{Conclusion}

We have developed a framework to generate $\mathrm{AE}$ signals using the impact information generated in DEM simulations. The AE signal model was developed based on particle-wall impact energy. By combining six modes of vibration, the model was able to reproduce the signals measured in the particle-plate impact experiments. The particle-wall collision data generated from DEM simulation of particle flow in a rotating drum was then recorded and passed to the $\mathrm{AE}$ model to generate the vibration signals on the drum surface. The feature peaks of the signals obtained through FFT analysis were able to link particle-wall impact energy, indicating that the $\mathrm{AE}$ signals can be used to characterise the internal flow of drums.

It should be noted, however, that the current work only simulated a simple system with mono-sized particles. For complicated systems with particle mixture, the relation between the signals and internal particle flow properties can be non-linear and requires further investigation. In addition, the simulated signals should be compared with the real signals from experiments before the framework is applied to real process monitors and optimisation.

The support from the Australia Research Council (ARC) ARC Research Hub on Computational Particle Technology (IH140100035) and Jiangsu Industrial Technology Research Institute (JITRI) is acknowledged. $\mathrm{YL}$ is also grateful to the Australian Government Research Training Program (RTP) Scholarship for financial support of his PhD study.

\section{References}

[1] B.A. Wills, J. Finch, Wills' mineral processing technology, Butterworth-Heinemann (2015)

[2] J. Tang, L. Zhao, J. Zhou, H. Yue, T. Chai, Miner. Eng. 23, $720(2010)$

[3] P. Huang, M. Jia, B. Zhong, Miner. Eng. 22, 1200 (2009)

[4] P. Hosseini, S. Martins, T. Martin, P. Radziszewski, F. Boyer, Miner. Eng. 24, 1440 (2011)

[5] J. Tang, J. Qiao, Z. Liu, X. Zhou, G. Yu, J. Zhao, Miner. Eng. 128, 294 (2018)

[6] Y. Zeng, K. Forssberg, Miner. Eng. 8, 4 (1995)

[7] J. Tang, T. Chai, W. Yu, L. Zhao, Control Eng. Pract. 20, 991 (2012)

[8] J. Tang, W. Yu, T. Chai, L. Zhao, Neurocomputing 82 , 167 (2012)

[9] J. Tang, J. Qiao, Z. Wu, T. Chai, J. Zhang, W. Yu, Mech. Syst. Signal Process 99, 142 (2018)

[10] L. McElroy, J. Bao, R. Yang, A. Yu, Powder Technol. 195, 177 (2009)

[11] L. McElroy, J. Bao, R. Yang, A. Yu, Powder Technol. 188, 234 (2009)

[12] H. Huang, J. Pan, P. McCormick, Int. J. Impact Eng. 19, 117 (1997)

[13] K. Asamene, L. Hudson, M. Sundaresan, Ultrasonics 59, 86 (2015)

[14] D. Buttle, C. Scruby, Wear 137, 63 (1990)

[15] Y. Hu, X. Huang, X. Qian, L. Gao, Y. Yan, Online particle size measurement through acoustic emission detection and signal analysis, IEEE Instrumentation and Measurement Technology Conference Proceedings (2014)

[16] T. Takahagi, M. Yokoi, M. Nakai, J. Acoust. Soc. Jpn. (E) 1,121 (1980)

[17] H. Zhu, Z. Zhou, R. Yang, A. Yu, Chem. Eng. Sci. 62, 3378 (2007)

[18] Y. Li, J. Bao, A. Yu, R. Yang, Chem. Eng. Sci. 231, $116251(2021)$ 